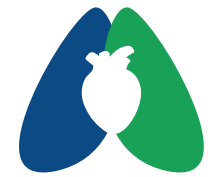

ASSOBRAFIR C I Ê N C I A

\section{Efeitos do treinamento muscular inspiratório na capacidade de exercício, força muscular inspiratória e qualidade de vida em indivíduos com insuficiência cardíaca: uma revisão sistemática}

\author{
Effects of inspiratory muscle training on exercise capacity, \\ inspiratory muscle strength and quality of life in individuals with \\ heart failure: a systematic review
}

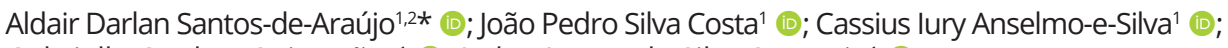
Gabriella Goulart Guimarães ${ }^{1}$ (1); Jadna Luana da Silva Sampaio ${ }^{1}$;

Túlio Mauro Silva Santos ${ }^{1}$ (D); Vinicius Minatel ${ }^{1}$ (D)

\begin{abstract}
Resumo
Introdução: A Insuficiência Cardíaca (IC) é uma patologia que cursa com redução da força muscular periférica e da musculatura respiratória culminando em redução da capacidade funcional e impactando negativamente na qualidade de vida. Por esta razão, as diretrizes atuais recomendam exercícios como terapia adjuvante ao tratamento farmacológico, dentre eles o treinamento muscular inspiratório (TMI). Objetivo: revisar sistematicamente a literatura sobre os efeitos do TMI com carga resistiva sobre a força muscular inspiratória, qualidade de vida e capacidade funcional de indivíduos com IC. Métodos: a busca sistemática foi conduzida através dos bancos de dados LILACS, PUBMED, SciELO, COCHRANE e PEDro. Para a busca, foram utilizados os seguintes termos MeSH: ("breathing exercises" AND "heart failure"; inspiratory muscle training" AND "heart failure") e ("treinamento muscular respiratório" AND "insuficiência cardíaca"). Foram incluídos ensaios clínicos, ensaios clínicos controlados e ensaios clínicos randomizados. Resultados: foram encontrados na busca 522 artigos. Ao final, apenas 13 estudos mostravam a utilização do TMI em indivíduos com IC de acordo com os critérios de elegibilidade. Desses, doze estudos relatam melhora na força muscular inspiratória $\left(\mathrm{PI}_{\max }\right)$, onze descrevem melhoria na capacidade funcional e sete na qualidade de vida. Conclusão: esta revisão sistemática apoia a ideia de que as intervenções de TMI com carga resistiva melhoram a $\mathrm{PI}_{\text {max }}$ a capacidade de exercício e a qualidade de vida em pacientes com IC. Adicionalmente, o TMI demonstra ser uma estratégia que merece ser considerada como intervenção adicional em pacientes com IC no que diz respeito a incremento significativos nessas variáveis.
\end{abstract}

Palavras-chave: Insuficiência Cardíaca; Treinamento Muscular Inspiratório; Qualidade de Vida; Capacidade Funcional; Força Muscular Inspiratória.

'Departamento de Fisioterapia, Centro Universitário Tiradentes (UNIT), Maceió, AL, Brasil

2Departamento de Fisioterapia, Universidade Federal de São Carlos (UFSCar), São Carlos, SP, Brasil

Como citar: Santos-de-Araújo AD, Costa JPS, Anselmo-e-Silva CI, Guimarães GG, Sampaio JLS, Santos TMS, et al. Efeitos do treinamento muscular inspiratório na capacidade de exercício, força muscular inspiratória e qualidade de vida em indivíduos com insuficiência cardíaca: uma revisão sistemática. ASSOBRAFIR Ciênc. 2022;13:e44675. https://doi. org/10.47066/21779333.AC.2020.0037

Submissão em: Outubro 05, 2021 Aceito em: Dezembro 21, 2021

Estudo realizado em: Centro Universitário Tiradentes (UNIT), Maceió, AL, Brasil. Aprovação ética: Não se aplica.

*Autor correspondente: Aldair Darlan Santos-de-Araújo.

E-mail: aldairdarlan1@gmail.com.

\begin{abstract}
Background: Heart failure (HF) is a pathology that courses with a reduction in peripheral muscle strength and respiratory muscles, culminating in a reduction in functional capacity and negatively impacting quality of life. For this reason, current guidelines recommend exercise as adjuvant therapy to pharmacological treatment, including inspiratory muscle training (IMT). Aim: to review systematically the literature on the effects of IMT with resistive load on inspiratory muscle strength, quality of life and functional capacity of individuals with HF. Methods: the systematic search for this review was conducted using the LILACS, PUBMED, SciELO, COCHRANE, and PEDro databases. For the search, the following MeSH terms were used: ("breathing exercises" AND "heart failure"; inspiratory muscle training" AND "heart failure") and ("respiratory muscle training" AND "heart failure"). Clinical studies, clinical trials, controlled clinical trials and randomized clinical trials were included. Results: 522 articles were found in the search. In the end, only 13 studies showed the use of IMT in individuals with HF according to the eligibility criteria. Of these, twelve studies report improvement in inspiratory muscle strength $\left(\mathrm{PI}_{\max }\right), 11$ describe improvement in functional capacity and seven in quality of life. Conclusion: this systematic review supports the idea that IMT interventions with resistive load improve $\mathrm{PI}_{\text {max }}$, exercise capacity and quality of life in patients with HF. Additionally, IMT demonstrates to be a strategy that deserves to be considered as an additional intervention in patients with HF with regard to significant increases in these variables.
\end{abstract}

Keywords: Heart Failure; Inspiratory Muscle Training; Quality of Life; Functional Capacity; Inspiratory Muscle Strength. 


\section{INTRODUÇÃO}

Nos últimos anos, a estimativa de novos casos diagnosticados de insuficiência cardíaca (IC) teve acréscimo de $12 \%{ }^{1}$. As projeções da American Heart Association apontam que mais de oito milhões de adultos serão diagnosticados com IC em 2030². Os altos custos sociais e a complexidade de atendimento a esses pacientes tornarão cada vez mais relevantes, assim como a necessidade de terapêuticas mais eficazes ${ }^{3}$. A IC é uma condição na qual o coração apresenta redução na capacidade de injetar sangue em quantidades necessárias para a manter a homeostase diante das demandas impostas ao organismo. Trata-se de uma patologia que cursa com redução da força muscular periférica e da musculatura respiratória culminando em redução da capacidade funcional e da qualidade de vida 4 .

Por esta razão, as diretrizes atuais recomendam exercícios e atividade física como terapias adjuvantes ao tratamento farmacológico ${ }^{5}$. O treinamento muscular respiratório é uma proposta eficaz na melhora da tolerância ao exercício associado à dispneia em pacientes com IC, incluindo os exercícios respiratórios e alternativas assistidas por equipamentos específicos ${ }^{6,7}$. O Treinamento Muscular Inspiratório (TMI) com resistência, por sua vez, consiste na realização de inspirações contra resistência através de diversos dispositivos. É considerado uma intervenção de fácil aplicação, baixo custo e clinicamente relevante no cenário da reabilitação, ganhando destaque entre as modalidades de intervenção para tratamento das disfunções da musculatura ventilatória e para melhora do desempenho. Através de dispositivos de resistência que oferecem diferentes níveis de carga linear ou alinear, o TMI pode promover aumento de força e resistência dos músculos ventilatórios e repercutir positivamente sobre a qualidade de vida e desempenho funcional ${ }^{8,9}$.

O TMI é apontado como terapia adjuvante no controle de sintomatologia e sinais limitantes em diversas patologias, demonstrando que seus efeitos sobre a força muscular inspiratória e resistência leva a melhora no consumo máximo de oxigênio $\left(\mathrm{VO}_{2 \mathrm{MAX}}\right)$, qualidade de vida e dispneia bem como da capacidade funcional ${ }^{10}$. Em ambiente hospitalar, o TMI auxilia pacientes com IC na melhora da $\mathrm{PI}_{\text {max' }}$ importante fator na melhora da força muscular, distância percorrida na marcha e dispneia ${ }^{11}$. Desfechos recentes apontam o TMI como estratégia eficaz para a melhora da capacidade ao exercício em pacientes com IC, justificado pela melhora no Teste de Caminhada de Seis minutos (TC6) ${ }^{12}$ e quando aplicado em alta intensidade, melhora o $\mathrm{VO}_{2 \mathrm{MAX}}$ nessa população ${ }^{13}$.

Revisões sistemáticas com alto rigor metodológico que discorrem sobre esse assunto já foram publicadas previamente, contudo, frequentemente novas literaturas baseadas em ensaios clínicos randomizados e controlados que abordem essa temática são lançadas, e frente à heterogeneidade dos protocolos bem como o número significativo de artigos que é publicado há uma necessidade de revisões sistemáticas atualizadas e que incluam os achados dos últimos anos. Diante disso, este estudo tem como objetivo revisar, analisar e atualizar sistematicamente a literatura sobre os efeitos do TMI com carga resistiva sem adição de exercícios adicionais sobre a força muscular inspiratória, qualidade de vida e capacidade funcional de indivíduos com IC.

\section{MÉTODOS}

Esta revisão sistemática foi conduzida de acordo com as orientações do Preferred Reporting Items for Systematic Reviews and Meta-Analyses (PRISMA) ${ }^{14}$ para o desenvolvimento de análise de revisões sistemáticas, sendo utilizado o programa Excel para Windows versão 2018 (Microsoft Corp, Washington, EUA) para auxiliar e sistematizar a busca e extração dos dados obtidos na busca das bases de dados eletrônicas. Ademais, a pesquisa foi estruturada com base na estratégia PICOS: P - paciente (indivíduos com insuficiência cardíaca), I - intervenção (treinamento muscular inspiratório), C - comparação (grupo controle, placebo ou outra intervenção), O desfechos (força muscular inspiratória, qualidade de vida e capacidade funcional), $\mathrm{S}$ - desenho dos estudos (ensaios clínicos, ensaios clínicos controlados e ensaios clínicos randomizados). O protocolo do estudo foi previamente registrado no International Prospective Register of Systematic Reviews (PROSPERO) sob o registro CRD42020199172.

\section{Estratégia de busca e fonte de dados}

A busca na literatura foi realizada nas bases de dados eletrônicas LILACS (Literatura Latino-americana e do Caribe em Ciências da Saúde), SciELO (Scientific Electronic Library Online), PubMED (via National Library of Medicine), Cochrane Central Register of Controlled Trials (COCHRANE) e Physiotherapy Evidence Database (PEDro). Para realizar a busca nestas bases de dados, foram definidos os seguintes termos MeSH para identificar a relação entre o treinamento muscular respiratório (breathing exercises; inspiratory muscle training) e os achados na insuficiência cardíaca (heart failure). Portanto, a partir da definição dos termos de busca foram adotadas as seguintes combinações dos termos selecionados ("breathing exercises" AND "heart failure"; "inspiratory muscle training" AND "heart failure") para realizar a busca as bases de dados LILACS, PubMED, COCHRANE, Scielo e PEDro; e ("treinamento muscular respiratório" AND "insuficiência cardíaca”) quando a busca foi feita na base de dados SciELO. Além das palavras chaves e termos descritos acima, a busca na literatura foi limitada a fim de incluir apenas estudos realizados com seres humanos e publicados em língua inglesa, portuguesa e espanhola. Adicionalmente, foram incluídos apenas ensaios clínicos, ensaios clínicos controlados e ensaios clínicos randomizados. A busca na literatura foi realizada em 29 de outubro de 2021. 


\section{Critérios de elegibilidade e exclusão}

Os critérios de elegibilidade para a seleção dos estudos foram: aqueles que descreviam a utilização do TMI sem adição de nenhuma outra intervenção em indivíduos com insuficiência cardíaca. Para tanto, foi levado em consideração os estudos que avaliaram o uso do TMI de maneira isolada e que compararam o efeito do treinamento com grupos controle, placebo ou outra intervenção. Os critérios de exclusão adotados foram: estudos pilotos, revisões, comunicações curtas, cartas, diretrizes, teses, dissertações, estudos qualitativos, resumos publicados em conferências nacionais e internacionais, estudos com experimento animal, estudos que não se apresentavam nos idiomas selecionados, além de artigos científicos que incluíram apenas indivíduos saudáveis.

\section{Critérios de seleção dos estudos}

O processo de seleção dos estudos a serem incluídos foi realizado a partir da análise de dois revisores independentes (ADSDA e GGG) seguindo os critérios de inclusão e exclusão descritos acima. Inicialmente, os estudos foram analisados levando em consideração o título e o resumo, e quando eles apresentassem potencial para elegibilidade eles eram selecionados para avaliação do texto na íntegra. No entanto, se houvesse discordância entre esses revisores, um terceiro revisor independente (JPSC) era consultado. Adicionalmente, os autores dos estudos eram contactados quando o artigo não estava disponível para o acesso na íntegra. Além disso, as referências dos artigos foram selecionadas a fim de buscar por outros estudos relevantes que pudessem compor a revisão.

\section{Extração dos dados dos estudos}

Foram extraídos de cada estudo as seguintes informações: a) características dos participantes e grupos (população, tamanho da amostra, grupos, gênero, idade e NYHA); b) intervenção, protocolo de exercícios e principais resultados.

\section{Avaliação da qualidade metodológica}

A qualidade metodológica dos estudos individuais foi avaliada utilizando a escala Physiotherapy Evidence Database (PEDro) ${ }^{15}$ e dois pesquisadores classificaram os artigos independentemente (ADSDA e JPCS). Quando houve alguma discordância, os pesquisadores discutiram o assunto para obter um consenso entre eles em relação à pontuação final.

A escala é composta por 11 itens relacionados a critérios metodológicos de ensaios clínicos randomizados. No entanto, a pontuação máxima é de 10 pontos, pois o primeiro item não recebe pontuação (especificando os critérios de elegibilidade). Os outros itens avaliados pela escala PEDro incluíram: alocação aleatória, alocação oculta, comparação inicial, participante cego, terapeuta cego, avaliador cego, medidas-chave de resultados $>85 \%$ dos participantes, intenção de tratar a análise, análise das comparações estatísticas intergrupos e medidas de variabilidade e precisão. O escore final foi agrupado em dois níveis: alta qualidade $(\geq 6)$ e baixa qualidade $(<6)$.

\section{RESULTADOS}

A partir da estratégia de busca foram encontrados quinhentos e dezessete estudos potencialmente relevantes identificados através de uma pesquisa nos bancos de dados (PubMed = 105; Scielo = 10; LILACS = 2; PEDro = 42; COCHRANE $=358$ ). Além disso, foram adicionados mais cinco artigos encontrados mediante análise das referências dos artigos incluídos neste estudo, totalizando 522 artigos. Destes, apenas treze estudos foram considerados elegíveis para análise de dados preenchendo os critérios de inclusão. O fluxograma de estudos por meio do processo de seleção se encontra apresentado na Figura 1.

Os treze estudos elegidos foram publicados entre 1998 e 2018. O tamanho das amostras variou de 18 a 59 participantes. O tamanho da amostra agrupada foi de 387, com uma média de 29 participantes por estudo. A média de idade variou entre 53 e 76 anos.

\section{Avaliação da qualidade metodológica}

O escore total da PEDro e a qualidade das evidências foram descritos na Tabela 1. Os estudos foram classificados como alta e baixa qualidade metodológica. A pontuação média da PEDro foi de 6 (variando de 3 a 8). Um total de 10 estudos foram classificados como alta qualidade metodológica16-25, enquanto que 3 estudos foram classificados como baixa qualidade metodológica ${ }^{26-28}$.

\section{Avaliação da capacidade de exercício}

Com relação à avaliação da capacidade de exercício foram utilizados o Teste de Exercício Cardiopulmonar (TECP), o Teste de Caminhada de 6 minutos (TC6) e o Teste de Caminhada de 12 minutos (TC12) como estratégia de obtenção de resultados dessa variável. Apenas um estudo ${ }^{26}$ utilizou o TC12 para avaliar a capacidade de exercício. 0 TECP consiste em submeter o indivíduo a um exercício de intensidade gradativamente crescente até a exaustão ou o surgimento de sintomas e/ou sinais limitantes com o intuito de avaliar os sistemas cardiovascular, respiratório, muscular e metabólico no esforço, sendo considerado padrão-ouro na avaliação funcional cardiorrespiratória ${ }^{29}$. Dentre as variáveis obtidas, o pico do volume de oxigênio $\left(\mathrm{VO}_{2}\right)$ demonstra ser uma das mais importantes. O pico de $\mathrm{VO}_{2}$ constitui o volume de oxigênio extraído do ar inspirado pela ventilação pulmonar num dado período de tempo, calculado, portanto, como a diferença entre o volume de oxigênio inspirado e expirado. 


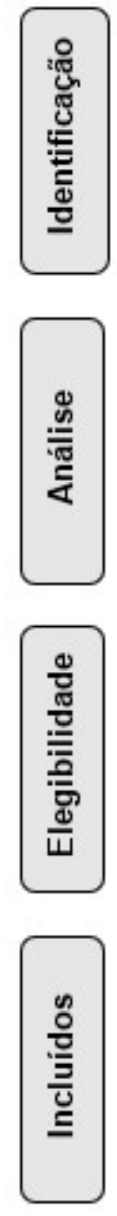

\section{Artigos identificados nas bases de dados $(n=517)$}

Artigos adicionados através de outras buscas $(n=5)$
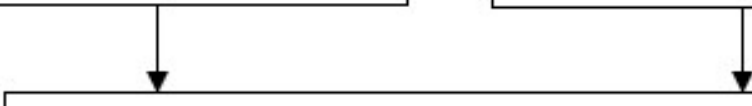

Artiqos após remocão dos duplicados $(n=231)$

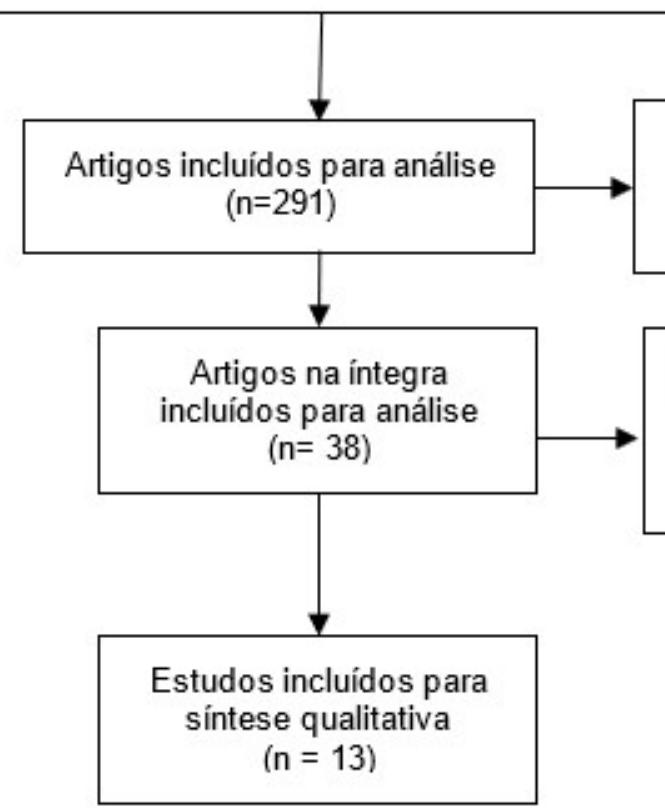

Artigos excluídos $(n=253)$

Não realizaram TMI

População diferente

Excluídos na íntegra $(\mathrm{n}=25)$

TMI associado a outra técnica

Sem carga resistiva Outros desfechos

Figura 1. Fluxograma da estratégia de busca e seleção dos artigos.

O TC12 foi desenvolvido inicialmente para avaliar o nível de condicionamento físico em soldados das forças armadas, sendo, portanto, chamado de Teste de Corrida de 12 minutos ${ }^{30}$. Mais tarde, McGavin et al..$^{31}$ o modificaram para Teste de Caminhada, com o intuito de avaliar a tolerância ao exercício em pacientes com bronquite crônica. Tendo em vista ser extenuante em pacientes debilitados, o mesmo foi adaptado para distâncias mais curtas, como é o caso do TC6. Esse, por sua vez, foi desenvolvido a fim de avaliar a capacidade funcional e estabelecer o prognóstico de pacientes com doenças cardiorrespiratórias ${ }^{32}$.

\section{Avaliação do treino muscular inspiratório}

No que diz respeito ao TMI, foram utilizados o threshold, o TRAINAIR e a válvula Orygen Dual como formas de intervenção nos estudos. O threshold consiste em um equipamento comercialmente disponibilizado na forma de um cilindro plástico transparente, contendo, em uma das extremidades, uma válvula que se mantém fechada pela pressão positiva de uma mola e, na outra extremidade, um bocal. A válvula bloqueia o fluxo aéreo até que o paciente gere pressão inspiratória suficiente para vencer a carga imposta pela mola, oferecendo uma resistência inspiratória ajustável de -7 a $-41 \mathrm{cmH}_{2} \mathrm{O}^{33,34}$.
O TrainAir é acompanhado de um software que permite feedback visual pelo indivíduo e apresenta sistematização de treinamento mais específico para endurance da musculatura inspiratória ${ }^{35}$. A Orygen Dual Valve consiste na união de dois modelos de dispositivos do tipo limiar de carga pressórica, cujo desenho de configuração tubular " $T$ " destina uma extremidade para treinamento muscular expiratório (TME) (carga mínima de $0 \mathrm{cmH}_{2} \mathrm{O}$ e máxima $60 \mathrm{cmH}_{2} \mathrm{O}$ ) e outra para TMI (carga mínima de $0 \mathrm{cmH}_{2} \mathrm{O}$ e máxima $90 \mathrm{cmH}_{2} \mathrm{O}$ ), ajuste a cada $1 \mathrm{cmH}_{2} \mathrm{O}$ e uma estrutura central para acoplamento do bocal. Corresponde a um dispositivo que permite TMI e/ou TME, portátil, de uso individual, do tipo limiar de carga pressórica com carga de mola, mecânico, dispensando o uso de fonte de energia para funcionamento ${ }^{36}$.

\section{Avaliação da qualidade de vida}

Quanto à qualidade de vida, foram utilizados os questionários Minnesota Living with Heart, traduzido e validado para a versão em português por Carvalho et al. ${ }^{37}$, e o SF-36, validado para a versão em português por Ciconelli et al. ${ }^{38}$. O primeiro consiste em uma importante ferramenta de avaliação da QV em pacientes com IC e fornece pontuações para duas dimensões, física e emocional. é composto por 21 questões relativas a 


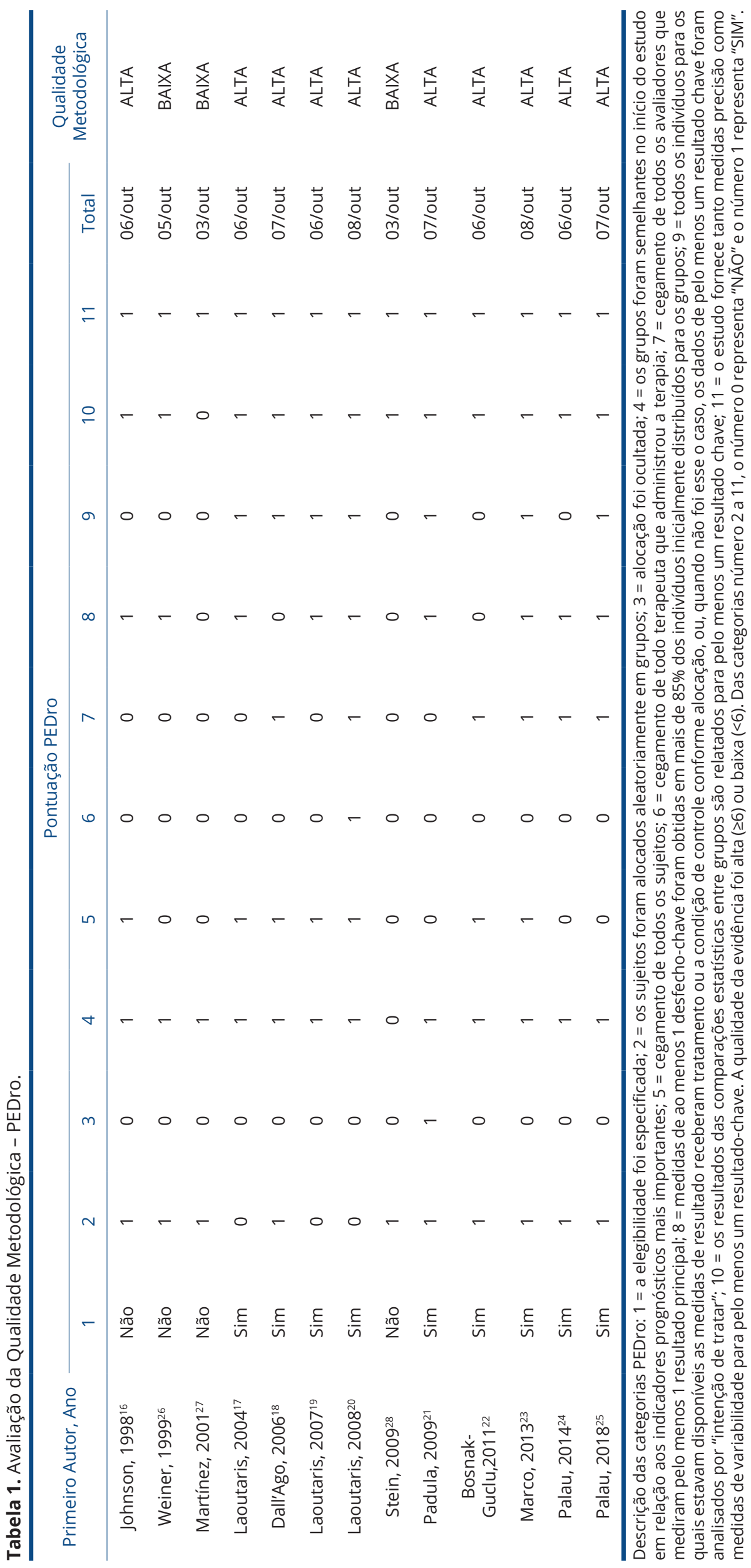


limitações que estão associadas com o quanto a IC impede os pacientes de viverem como gostariam. Por sua vez, o SF-36 consta de 11 questões e 36 itens que englobam oito componentes, representados por capacidade funcional, aspectos físicos, dor, estado geral da saúde, vitalidade, aspectos sociais, aspectos emocionais, saúde mental e saúde atual e dos últimos 12 meses. O indivíduo recebe um escore em cada domínio, que varia de 0 a 100 , sendo 0 o pior escore e 100, o melhor.

\section{Características dos participantes}

As características dos participantes e dos grupos foram descritas em Tabela 2. Em relação aos pacientes que apresentavam IC com fração de ejeção preservada, foram conduzidos dois estudos com pacientes que apresentavam classe funcional de acordo com a NYHA $\geq \|^{24,25}$. Um estudo foi desenhado para uma população de IC com fraqueza muscular inspiratória, e o mesmo não identificou qual classe funcional os participantes deveriam estar para serem incluídos nos estudos ${ }^{18}$.

Tabela 2. Características dos estudos incluídos nesta revisão.

\begin{tabular}{|c|c|c|c|c|c|}
\hline \multirow{2}{*}{ ESTUDO } & \multirow{2}{*}{ POPULAÇÃO } & \multirow{2}{*}{$\begin{array}{c}\text { GRUPO E } \\
\text { TAMANHO DA } \\
\text { AMOSTRA }\end{array}$} & \multirow{2}{*}{ NYHA } & \multirow{2}{*}{ SEXO (M/F) } & IDADE \\
\hline & & & & & (ANOS) \\
\hline \multirow{2}{*}{ Johnson, $1998^{16}$} & \multirow{2}{*}{$\begin{array}{l}\text { Insuficiência Cardíaca } \\
\text { Crônica Estável }\end{array}$} & & \multirow{2}{*}{$I I-I I I$} & \multirow{2}{*}{ 15/mar } & GC $(63,4 \pm 4,5)$ \\
\hline & & $\mathrm{Gl}(\mathrm{n}=9)$ & & & $\mathrm{Gl}(70 \pm 4,6)$ \\
\hline \multirow{2}{*}{ Weiner, $1999^{26}$} & \multirow{2}{*}{$\begin{array}{c}\text { Insuficiência Cardíaca } \\
\text { Congestiva }\end{array}$} & $G C(n=10)$ & \multirow{2}{*}{$\|-I I I$} & \multirow{2}{*}{ NR } & $\mathrm{GC}(63,8 \pm 4)$ \\
\hline & & $\mathrm{Gl}(\mathrm{n}=10)$ & & & GI $(66,2 \pm 4,6)$ \\
\hline \multirow{2}{*}{ Martínez, 200127 } & \multirow{2}{*}{$\begin{array}{c}\text { Insuficiência Cardíaca } \\
\text { Crônica }\end{array}$} & $G C(n=11)$ & \multirow{2}{*}{$\|-111$} & \multirow{2}{*}{ 16/abr } & $\mathrm{GC}(57 \pm 13)$ \\
\hline & & $\mathrm{Gl}(n=9)$ & & & GI $(60 \pm 14)$ \\
\hline \multirow[b]{2}{*}{ Laoutaris, $2004^{17}$} & Insuficiência Cardíaca & $G C(n=15)$ & \multirow[b]{2}{*}{$I I-I I I$} & \multirow[b]{2}{*}{$31 / 4$} & $\mathrm{GC}(60 \pm 2,6)$ \\
\hline & $\begin{array}{c}\text { Crônica Moderada a } \\
\text { Grave }\end{array}$ & $\mathrm{Gl}(\mathrm{n}=20)$ & & & GI $(57,6 \pm 2,3)$ \\
\hline \multirow[b]{2}{*}{ Dall'Ago, $2006^{18}$} & Insuficiência Cardíaca & $\mathrm{GC}(n=16)$ & \multirow[b]{2}{*}{ NR } & \multirow[b]{2}{*}{$17 / 15$} & $\mathrm{GC}(58 \pm 2)$ \\
\hline & Crônica + Fraqueza & & & & Gl $(54+3)$ \\
\hline \multirow{3}{*}{ Laoutaris, $2007^{19}$} & \multirow{3}{*}{$\begin{array}{c}\text { Insuficiência Cardíaca } \\
\text { Crônica }\end{array}$} & & & \multirow{3}{*}{$32 / 6$} & \\
\hline & & GC (n=23) & \multirow{2}{*}{$I I-I I I$} & & $\mathrm{GC}(59 \pm 2)$ \\
\hline & & $\mathrm{GI}(\mathrm{n}=15)$ & & & GI $(53 \pm 2)$ \\
\hline \multirow{2}{*}{ Laoutaris, $2008^{20}$} & \multirow{2}{*}{$\begin{array}{l}\text { Insuficiência Cardíaca } \\
\text { Crônica. }\end{array}$} & $\mathrm{GC}(\mathrm{n}=9)$ & \multirow{2}{*}{ 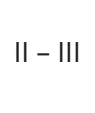 } & \multirow{2}{*}{ 20/mar } & $\mathrm{GC}(57,3 \pm 4)$ \\
\hline & & GI $(n=14)$ & & & GI $(53,4 \pm 2,1)$ \\
\hline & Insuficiência Cardíaca & $\mathrm{GC}(\mathrm{n}=16)$ & & & GC: NR \\
\hline Stein, $2009^{28}$ & Crônica & $\mathrm{GI}(\mathrm{n}=16)$ & NR & NR & GI: NR \\
\hline Padula 200921 & Insuficiência Cardíaca & $G C(n=17)$ & ॥॥ & dez/20 & $\mathrm{GC}(73 \pm N R)$ \\
\hline 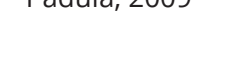 & Crônica & GI $(n=15)$ & IIIIII & UeZl $\angle U$ & GI $(76 \pm N R)$ \\
\hline Bosnak- & Insuficiência Cardíaca & $G C(n=14)$ & $\|-\|$ & 24/iun & GC $(65,71 \pm 10,52)$ \\
\hline Guclu,201122 & 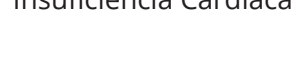 & GI $(n=16)$ & $\pi-m$ & $24 /$ Jun & $\mathrm{Gl}(69,50 \pm 7,96)$ \\
\hline & Insuficiência Cardíaca & $\mathrm{GC}(\mathrm{n}=11)$ & $\|$ - ॥ & 17/mai & $\mathrm{GC}(70,1 \pm 10,75)$ \\
\hline Marco, $2013^{25}$ & Crônica & $\mathrm{GI}(\mathrm{n}=11)$ & $11-111$ & 1//mal & GI $(68,5 \pm 8,88)$ \\
\hline & Insuficiência Cardíaca & $G C(n=12)$ & & & $\mathrm{GC}(68 \pm 8)$ \\
\hline Palau, $2014^{24}$ & $\begin{array}{c}\text { com Fração de Ejeção } \\
\text { Preservada }\end{array}$ & $\mathrm{GI}(\mathrm{n}=14)$ & $\geq \mathrm{II}$ & $13 / 13$ & $\mathrm{Gl}(74 \pm 3)$ \\
\hline & & $G C(n=13)$ & & & GC $(75 \pm 9)$ \\
\hline & Insuficiência Cardíaca & GI: TMI (n=15) & & & GI: TMI $(75 \pm 10)$ \\
\hline Palau, $2018^{25}$ & $\begin{array}{c}\text { com Fração de Ejeção } \\
\text { Preservada. }\end{array}$ & Gl: $E F(n=15)$ & $\geq \mathrm{II}$ & $25 / 34$ & $\mathrm{EF}(72 \pm 9)$ \\
\hline & & GI: EF+TMI $(n=16)$ & & & $E F+T M I(73 \pm 10)$ \\
\hline
\end{tabular}

Dados apresentados como: média, média desvio padrão. GC: grupo controle; Gl: grupo intervenção; TMl: treino muscular inspiratório; EF: eletroestimulação funcional; NYHA: New York Heart Association; NR: não relatado. 
Apenas um estudo foi conduzido em pacientes com IC crônica de moderava a grave com classe funcional NYHA II-III ${ }^{17}$. Enquanto outro foi delineado em indivíduos com IC congestiva e classificação funcional NYHA $\|-||{ }^{26}$. Todos os outros estudos, num total de oito, foram conduzidos com indivíduos com IC crônica e classe funcional NYHA IIIII16,19-22,27, com exceção de Stein et al. ${ }^{28}$ que não descreveu qual classe funcional pertencia os indivíduos incluídos em seu estudo.

\section{Características das intervenções}

As intervenções foram descritas na Tabela 3. O regime de treino foi amplamente baseado em uma certa porcentagem da $\mathrm{PI}_{\max }$. A carga resistiva utilizada no grupo intervenção dos estudos incluídos variaram em um percentual de $25-60 \%$ da $\mathrm{PI}_{\max }$. Um estudo utilizou um dispositivo de treinamento em um modelo protótipo de válvula Orygen-Dual, com uma carga de 10 repetições máximas consecutivas ajustados semanalmente ${ }^{23}$. Para o grupo controle, a intensidade do treinamento variou de sem carga a $20 \%$ de $\mathrm{PI}_{\text {max }}$.

O período total de treinamento variou de 4 a 12 semanas e a duração do treinamento em cada sessão variou de 5 a 30 minutos, 1 a 3 vezes por dia, de 3 a 7 vezes por semana. A maioria desses estudos utilizou o Threshold como dispositivo para o TMI, com exceção de um estudo cujo o dispositivo de treinamento era um protótipo da válvula Orygen-Dual23 e três estudos que avaliaram a resistência muscular respiratória por meio de um dispositivo de biofeedback controlado por computador (TRAINAIR, Project Electronics Ltd., Kent, Reino Unido) ${ }^{17,19,20}$.

\section{TMI versus força muscular inspiratória $\left(\mathrm{PI}_{\max }\right)$}

As respostas da relação entre o TMI e a IC estão apresentadas na Tabela 3. No que diz respeito aos resultados do TMI na $\mathrm{PI}_{\text {max' }}$ todos os estudos avaliaram o efeito do mesmo sobre essa variável, com exceção de Palau et al. ${ }^{25}$, cujo o mesmo não avaliou esse desfecho. Os resultados demonstraram ser favoráveis, gerando incremento significante $(p<0,05)$ em todos os estudos quando comparados aos valores basais.

\section{TMI versus capacidade funcional}

Quanto à capacidade funcional, dois estudos não avaliaram a influência da TMI sobre essa variável21,23. No entanto, para todos os outros estudos, um total de onze, os resultados se mostraram favoráveis e significantes ( $p$ $<0,05)$, com exceção do estudo conduzido por Jonhson, Cowley e Kinnear ${ }^{27}$ que não conseguiram encontrar um resultado significativo após a aplicação do protocolo de $\mathrm{TMI}$, chegando à conclusão de que o mesmo não resulta em melhorias significativas na tolerância ao exercício. Os resultados estão expressos na Tabela 3.

\section{TMI versus qualidade de vida}

A respeito do TMI sobre a qualidade de vida, seis estudos não avaliaram esse desfecho ${ }^{19-21,26-28}$. Dois estudos não encontraram resultados significativos ${ }^{16,23}$. Todos os outros estudos, um total de sete, demonstraram resultados com diferença estatística significante quando comparado os valores basais aos valores resultantes após o protocolo de TMI $(p<0,05)$. Os resultados estão expressos na Tabela 3.

\section{DISCUSSÃO}

Esta revisão sistemática, por meio de uma avaliação de 13 ensaios clínicos, conduzida com o intuito de determinar os efeitos do TMI com adição de carga na força muscular inspiratória, capacidade funcional e qualidade de vida de indivíduos com IC, demonstrou que o TMI pode ser uma opção de treinamento capaz de gerar incrementos positivos nessas variáveis na população em questão. Os efeitos positivos do $\mathrm{TMI}$ na $\mathrm{PI}_{\max }$ foram observados em todos os artigos, exceto Palau et al. ${ }^{25}$ que não avaliou essa variável. A despeito da capacidade funcional, a maioria dos estudos incluídos nesta revisão indicaram melhora nessa variável, com exceção de Johnson, Cowley e Kinear ${ }^{16}$ que não encontrou diferença significativa após a aplicação do protocolo de treinamento desenhado para seu estudo. Com relação à qualidade de vida, apenas sete estudos encontram resultados significativos, dois relataram não haver melhora significante ${ }^{16,23}$. Ademais, seis estudos não avaliaram a mesma 19-21,26-28.

A literatura tem demonstrado que essa população apresenta força muscular respiratória prejudicada $22,39-42$. Os pacientes que cursam com comprometimento da função respiratória e cardíaca apresentam disfunções capazes de levar à intolerância ao exercício, e isso se justifica frente à responsabilidade que esses sistemas conferem à manutenção e funcionalidade adequada da função respiratória normal, com capacidade de captação e distribuição de oxigênio aos tecidos metabolicamente ativos ${ }^{41}$. Um aspecto relevante nesta revisão diz respeito à carga analisada nos artigos que variou entre $25-60 \%$ da $\mathrm{PI}_{\max }$ no grupo intervenção. Para a obtenção de tais resultados nas variáveis acimas citadas diversas estratégias de aplicação do TMI foram usadas, levando à conclusão de que tais alcances dependam, portanto, da carga, da frequência e da duração da intervenção.

Sugere-se que essa faixa de carga no TMI ofereça maior segurança aos indivíduos que apresentam doenças cardiovasculares, tendo em vista que, quando realizado em intensidades elevadas esse tipo de treinamento possa causar fadiga precoce na musculatura respiratória e aumento da atividade neural simpática capaz de desencadear aumento no trabalho do miocárdio, resistência vascular periférica e pressão arterial, colocando essa população a uma exposição maior de eventos cardiovasculares agudos ${ }^{43,44}$. 


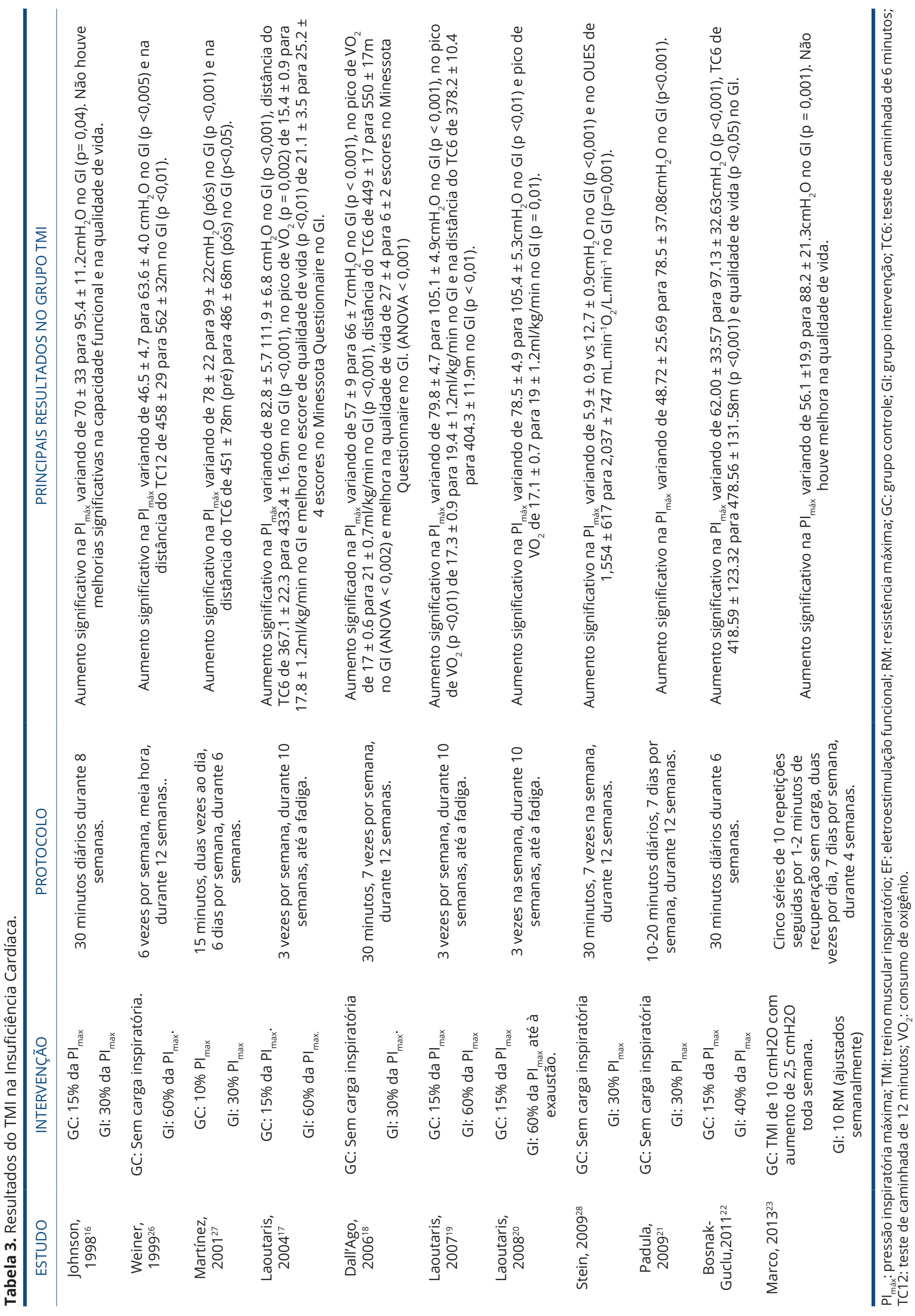


Efeitos do treinamento muscular inspiratório na capacidade de exercício, força muscular inspiratória e qualidade de vida em indivíduos com insuficiência cardíaca: uma revisão sistemática.

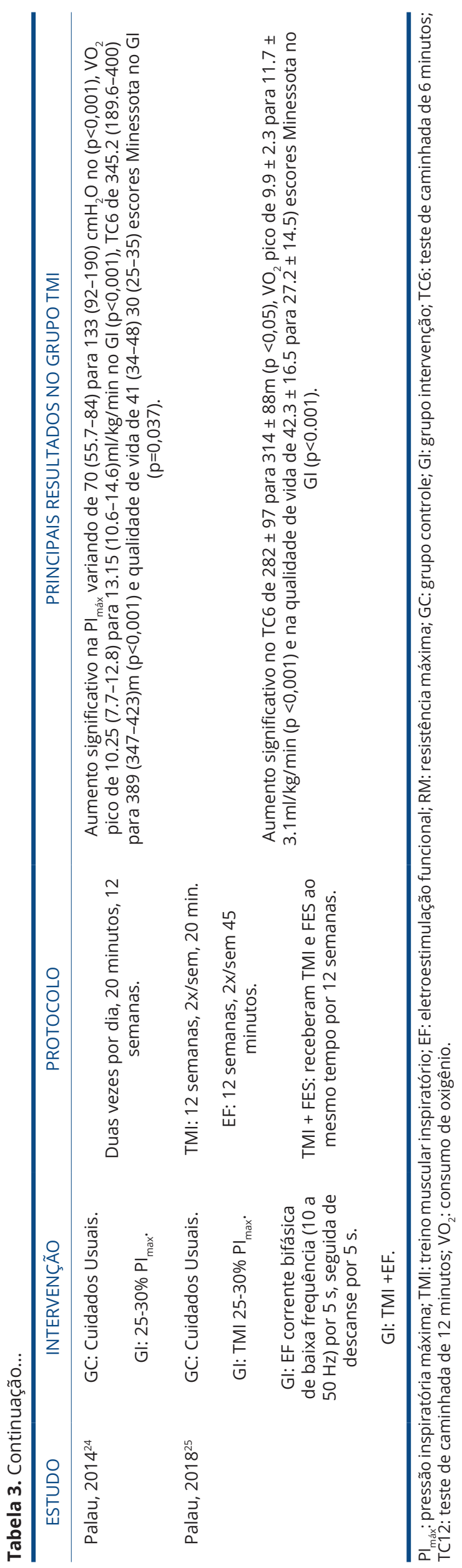


A capacidade funcional de indivíduos com IC apresentase comprometida ${ }^{45,46}$. Resumidamente, isso se deve à sintomatologia apresentada durante o esforço, tais como a presença de fadiga e dispneia, o que dificulta a realização das atividades de vida diária, estando diretamente relacionada à fraqueza da musculatura respiratória ${ }^{47,48}$. Frente a esse cenário, algumas estratégias que culminam na avaliação da capacidade funcional, como o TC6 e o TECP, tem sido utilizada. Adicionalmente, o TMI surge como um método de intervenção em pacientes com IC com resposta positiva sobre a tolerância ao exercício.

Neste estudo, os resultados da capacidade funcional estão em consonância com a literatura, apontando benefícios significativos do uso do TMI sobre essa variável. Apenas um estudo ${ }^{16}$ não encontrou resposta significativa após o uso de um protocolo de TMI: 30 minutos diários, resistência de $30 \%$ da $\mathrm{PI}_{\text {max' }}$ durante 8 semanas. Coincidentemente, é um estudo que apresenta um pequeno número de participantes. Dall'Ago et al. ${ }^{18}$ e Bosnak-Guclu et al. ${ }^{22}$ utilizaram protocolos similares, utilizando TMI durante 30 minutos diariamente, no entanto, o primeiro aplicou durante seis semanas com uma resistência maior, no valor de $40 \%$ da $\mathrm{PI}_{\text {max' }}$ enquanto que o segundo aplicou um protocolo durante doze semanas com resistência da $\mathrm{PI}_{\max }$ de $30 \%$, ambos apresentando incremento significativo da capacidade funcional. A análise desses dois estudos com protocolos similares leva a acreditar que o número de semanas ou a carga de resistência da $\mathrm{PI}_{\max }$ possam influenciar na resposta com resultados significativos nessa variável.

Possivelmente, o incremento na capacidade funcional observada pode estar relacionado ao atraso no desenvolvimento da fadiga diafragmática, aumento da eficiência ventilatória, redução da atividade mediada pelo sistema nervoso autônomo simpático, redução da liberação de produtos metabólitos, melhora da vasodilatação sistêmica e maior perfusão dos músculos periféricos ${ }^{10,49}$.

Indivíduos portadores de IC cursam com qualidade de vida afetada em detrimento dos sinais e sintomas intrínsecos à patologia ${ }^{50,51}$. Nossos resultados mostram haver melhora da qualidade de vida após um protocolo de TMI, no entanto, dois autores encontraram resultados divergentes, apontando não haver incremento significativo nessa variável ${ }^{16,23}$. Marco et al. ${ }^{23}$ é o único estudo no qual o protocolo de TMI foi aplicado durante apenas quatro semanas, um pequeno espaço de tempo quando comparado aos outros estudos, podendo, sobretudo, denotar um aspecto que que possivelmente tenha interferido nesse resultado. Além disso, o mesmo aponta em seus resultados que o estudo se trata de um número de participantes pequeno e com pontuações basais relativamente boas apresentadas na aplicação do questionário, sugerindo ser interessante repetir essa análise em um grupo de pacientes mais sintomáticos. Por sua vez, Johnson, Cowley e Kinnear ${ }^{16}$ relaciona a não melhoria da qualidade de vida de seus participantes a algumas vertentes: 1) o TMI não foi intensivo o suficiente; 2 ) a porcentagem de $\mathrm{PI}_{\max }$ utilizada possivelmente deveria ser maior; 3 ) os indivíduos do grupo treinamento apresentavam $\mathrm{PI}_{\max }$ menor que $\mathrm{o}$ grupo controle, e o valor final (pós-treinamento) entre os dois grupos foi similar.

Em geral, a qualidade metodológica dos artigos incluídos variou de 3 a 8 pontos na escala PEDro. De maneira resumida, os estudos de baixa qualidade somam um número de três, enquanto 10 estudos apresentam alta qualidade metodológica. Os principais itens não relatados nos estudos foram: alocação oculta e cegamento do terapeuta que administrou a terapia (doze artigos). Embora os resultados oferecidos pela escala PEDro não avaliem a validade externa do estudo, nem a magnitude do efeito de tratamento (isto é, se os resultados são clinicamente relevantes ou não), o alto rigor metodológico da maioria dos estudos aqui incluídos e a qualidade da evidência podem contribuir para o critério de tomada de decisão clínica, podendo proporcionar conclusões com boa validade científica e escolha do método para compor as medidas de tratamentos adotadas nessa população.

Essa revisão sistemática se limita frente a alguns aspectos: a) presença de diferentes tipos de protocolos de TMI. Embora tenhamos incluídos protocolos de TMI com carga, existiu uma alta heterogeneidade dos estudos incluídos quanto aos protocolos em termos de duração, frequência, intensidade e tempo de intervenção; b) diferentes graus de gravidade da patologia; c) diferentes dispositivos de treinamento utilizados; d) baixa quantidade de bases de dados selecionadas para a busca dos estudos.

\section{CONCLUSÃO}

Esta revisão sistemática apoia a ideia de que as intervenções de TMI com carga resistiva melhoram a $\mathrm{PI}_{\text {max }}$ a capacidade de exercício e a qualidade de vida em pacientes com IC. Os resultados oferecem à equipe clínica insights práticos e estratégias terapêuticas práticas baseadas em configurações para melhorar a condição de pacientes com IC. Adicionalmente, o TMI demonstra ser uma estratégia que merece ser considerada como intervenção adicional nessa população no que diz respeito a incremento significativos nessas variáveis.

\section{FONTE DE FINANCIAMENTO}

Nada a declarar.

\section{CONFLITO DE INTERESSES}

Nada a declarar. 


\section{REFERÊNCIAS}

1. Conrad N, Judge A, Tran J, Mohseni H, Hedgecott D, Crespillo $A P$, et al. Temporal trends and patterns in heart failure incidence: a population-based study of 4 million individuals. Lancet. 2018;391(10120):572-80. http://dx.doi.org/10.1016/ S0140-6736(17)32520-5. PMid:29174292.

2. Benjamin EJ, Virani SS, Callaway CW, Chamberlain AM, Chang AR, Cheng $S$, et al. Heart disease and stroke statistics-2018 update: a report from the American Heart Association. Circulation. 2018;137(12):e67-492. http://dx.doi. org/10.1161/CIR.0000000000000558. PMid:29386200.

3. Wang $\mathrm{MH}$, Yeh ML. Respiratory training interventions improve health status of heart failure patients: a systematic review and network meta-analysis of randomized controlled trials. World J Clin Cases. 2019;7(18):2760-75. http://dx.doi. org/10.12998/wjcc.v7.i18.2760. PMid:31616691.

4. Nogueira IDB, Nogueira PAMS, Vieira RHG, Souza RJS, Coutinho AE, Ferreira GMH. Capacidade funcional, força muscular e qualidade de vida na insuficiência cardíaca. Rev Bras Med Esporte. 2017;23(3):184-8. http://dx.doi. org/10.1590/1517-869220172303162427.

5. Ponikowski P, Voors AA, Anker SD, Bueno H, Cleland JGF, Coats AJS, et al. 2016 ESC Guidelines for the diagnosis and treatment of acute and chronic heart failure: the Task Force for the diagnosis and treatment of acute and chronic heart failure of the European Society of Cardiology (ESC) developed with the special contribution of the Heart Failure Association (HFA) of the ESC. Eur Heart J. 2016;37(27):2129-200. http:// dx.doi.org/10.1093/eurheartj/ehw128. PMid:27206819.

6. Cahalin LP, Arena R, Guazzi M, Myers J, Cipriano G, Chiappa $\mathrm{G}$, et al. Inspiratory muscle training in heart disease and heart failure: a review of the literature with a focus on method of training and outcomes. Expert Rev Cardiovasc Ther. 2013;11(2):161-77. http://dx.doi.org/10.1586/ erc.12.191. PMid:23405838.

7. Cahalin LP, Arena RA. Breathing exercises and inspiratory muscle training in heart failure. Heart Fail Clin. 2015;11(1):149-72. http://dx.doi.org/10.1016/j. hfc.2014.09.002. PMid:25432483.

8. Hermes BM, Cardoso DM, Gomes TJ, Santos TD, Vicente MS, Pereira SN, et al. Short-term inspiratory muscle training potentiates the benefits of aerobic and resistance training in patients undergoing CABG in phase II cardiac rehabilitation program. Rev Bras Cir Cardiovasc. 2015;30(4):474-81. http:// dx.doi.org/10.5935/1678-9741.20150043. PMid:27163422.

9. Pazzianotto-Forti EM, Munno CMC, Merino DFB, Rocha MRS, Mori TA, Rasera IJr. Effects of inspiratory exercise with linear and nonlinear load on respiratory variables post-bariatric surgery. Respir Care. 2019;64(12):1516-22. http://dx.doi. org/10.4187/respcare.05841. PMid:31387894.

10. Plentz RDM, Sbruzzi G, Ribeiro RA, Ferreira JB, Dal Lago P. Treinamento muscular inspiratório em pacientes com insuficiência cardíaca: metanálise de estudos randomizados. Arq Bras Cardiol. 2012;99(2):762-71. http://dx.doi.org/10.1590/S0066-782X2012001100011. PMid:22964897.

11. Sadek Z, Salami A, Joumaa WH, Awada C, Ahmaidi S, Ramadan W. Best mode of inspiratory muscle training in heart failure patients: a systematic review and metaanalysis. Eur J Prev Cardiol. 2018;25(16):1691-701. http:// dx.doi.org/10.1177/2047487318792315. PMid:30073849.

12. Wu J, Kuang L, Fu L. Effects of inspiratory muscle training in chronic heart failure patients: a systematic review and meta-analysis. Congenit Heart Dis. 2018;13(2):194-202. http://dx.doi.org/10.1111/chd.12586. PMid:29423938.

13. Gomes M No, Ferrari F, Helal L, Lopes AA, Carvalho VO, Stein $\mathrm{R}$. The impact of high-intensity inspiratory muscle training on exercise capacity and inspiratory muscle strength in heart failure with reduced ejection fraction: a systematic review and meta-analysis. Clin Rehabil. 2018;32(11):1482-92. http:// dx.doi.org/10.1177/0269215518784345. PMid:29984598.

14. Page MJ, Mckenzie JE, Bossuyt PM, Boutron I, Hoffmann TC, Mulrow CD, et al. The PRISMA 2020 statement: an updated guideline for reporting systematic reviews. BMJ. 2021;372(71):n71. http://dx.doi.org/10.1136/bmj.n71. PMid:33782057.

15. Moseley AM, Herbert RD, Sherrington C, Maher CG. Evidence for physiotherapy practice: a survey of the Physiotherapy Evidence Database (PEDro). Aust J Physiother. 2002;48(1):439. http://dx.doi.org/10.1016/S0004-9514(14)60281-6. PMid:11869164.

16. Johnson PH, Cowley AJ, Kinnear WJ. A randomized controlled trial of inspiratory muscle training in stable chronic heart failure. Eur Heart J. 1998;19(8):1249-53. http://dx.doi. org/10.1053/euhj.1998.1024. PMid:9740347.

17. Laoutaris I, Dritsas A, Brown MD, Manginas A, Alivizatos PA, Cokkinos DV. Inspiratory muscle training using an incremental endurance test alleviates dyspnea and improves functional status in patients with chronic heart failure. Eur J Cardiovasc Prev Rehabil. 2004;11(6):489-96. http://dx.doi.org/10.1097/01.hjr.0000152242.51327.63. PMid:15580060.

18. Dall'Ago P, Chiappa GR, Guths $H$, Stein R, Ribeiro JP. Inspiratory muscle training in patients with heart failure and inspiratory muscle weakness: a randomized trial. J Am Coll Cardiol. 2006;47(4):757-63. http://dx.doi.org/10.1016/j. jacc.2005.09.052. PMid:16487841.

19. Laoutaris ID, Dritsas A, Brown MD, Manginas A, Kallistratos MS, Degiannis $D$, et al. Immune response to inspiratory muscle training in patients with chronic heart failure. Eur J Cardiovasc Prev Rehabil. 2007;14(5):679-85. http://dx.doi. org/10.1097/HJR.0b013e3281338394. PMid:17925628.

20. Laoutaris ID, Dritsas A, Brown MD, Manginas A, Kallistratos MS, Chaidaroglou A, et al. Effects of inspiratory muscle training on autonomic activity, endothelial vasodilator function, and $\mathrm{N}$-terminal pro-brain natriuretic peptide levels in chronic heart failure. J Cardiopulm Rehabil Prev. 2008;28(2):99-106. http://dx.doi.org/10.1097/01. HCR.0000314203.09676.b9. PMid:18360185.

21. Padula CA, Yeaw E, Mistry S. A home-based nurse-coached inspiratory muscle training intervention in heart failure. Appl Nurs Res. 2009;22(1):18-25. http://dx.doi.org/10.1016/j. apnr.2007.02.002. PMid:19171291.

22. Bosnak-Guclu M, Arikan H, Savci S, Inal-Ince D, Tulumen $\mathrm{E}$, Aytemir K, et al. Effects of inspiratory muscle training in patients with heart failure. Respir Med. 2011;105(11):167181. http://dx.doi.org/10.1016/j.rmed.2011.05.001. PMid:21621993.

23. Marco E, Ramírez-Sarmento AL, Coloma A, Sartor M, CominColet J, Vila J, et al. High-intensity vs. sham inspiratory muscle training in patients with chronic heart failure: a prospective randomized trial. Eur J Heart Fail. 2013;15(8):892-901.

24. Palau $P$, Domínguez $E$, Núñez $E$, Schmid JP, Vergara $P$, Ramón JM, et al. Effects of inspiratory muscle training in patients with heart failure with preserved ejection fraction. Eur J Prev Cardiol. 2014;21(12):1465-73. http://dx.doi. org/10.1177/2047487313498832. PMid:23864363.

25. Palau P, Domínguez E, López L, Ramón JM, Heredia R, González J, et al. Inspiratory muscle training and functional electrical stimulation for treatment of heart failure with preserved ejection fraction: the TRAINING-HF Trial. Rev Esp Cardiol. 2019;72(4):288-97. http://dx.doi.org/10.1016/j. rec.2018.01.010. PMid:29551699.

26. Weiner P, Waizman J, Magadle R, Berar-Yanay N, Pelled B. The effect of specific inspiratory muscle training on the sensation 
of dyspnea and exercise tolerance in patients with congestive heart failure. Clin Cardiol. 1999;22(11):727-732. http://dx.doi. org/10.1002/clc.4960221110.

27. Martínez A, Lisboa C, Jalil J, Muñoz V, Díaz O, Casanegra P, et al. Entrenamiento selectivo de los músculos respiratorios en pacientes con insuficiencia cardíaca crónica. Rev Med Chil. 2001;129(2):133-9. http://dx.doi.org/10.4067/S003498872001000200002. PMid:11351463.

28. Stein R, Chiappa GR, Guths H, Dall'Ago P, Ribeiro JP. Inspiratory muscle training improves oxygen uptake efficiency slope in patients with chronic heart failure. Cardiopulm Rehabil Prev. 2009;29(6):392-5. http://dx.doi. org/10.1097/HCR.0b013e3181b4cc41. PMid:19809347.

29. Herdy AH, Ritt LEF, Stein R, Araújo CGS, Milani M, Meneghelo RS. Teste cardiopulmonar de exercício: fundamentos, aplicabilidade e interpretação. Arq Bras Cardiol. 2016;107(5):467-81. http://dx.doi.org/10.5935/ abc.20160171. PMid:27982272.

30. Cooper DM, Kaplan MR, Baumgarten L, Weiler-Ravell D, Whipp BJ, Wasserman K. Coupling of ventilation and CO2 production during exercise in children. Pediatr Res. 1987;21(6):568-72. http://dx.doi.org/10.1203/00006450$198706000-00012$.

31. McGavin CR, Artvinli M, Naoe H, McHardy GJ. Dyspnoea, disability, and distance walked: comparison of estimates of exercise performance in respiratory disease. BMJ. 1978;2(6132):241-3. http://dx.doi.org/10.1136/ bmj.2.6132.241. PMid:678885.

32. Dourado VR. Equações de referência para o teste de caminhada de seis minutos em indivíduos saudáveis. Arq Bras Cardiol. 2011;96(6):1-11. http://dx.doi.org/10.1590/ S0066-782X2011005000024.https://www.ncbi.nlm.nih. gov/entrez/query.fcgi?cmd=Retrieve\& $\mathrm{db}=$ PubMed\&lis t_uids $=22262150 \&$ dopt $=$ Abstract

33. Fonseca MA, Cader SA, Dantas EHM, Bacelar SC, Silva EB, Leal SM. Programas de treinamento muscular respiratório: impacto na autonomia funcional de idosos. Rev Assoc Med Bras. 2010;56(6):642-8. http://dx.doi.org/10.1590/S010442302010000600010. PMid:21271128.

34. Trevisan ME, Porto AS, Pinheiro TM. Influência do treinamento da musculatura respiratória e de membros inferiores no desempenho funcional de indivíduos com DPOC. Fisioter Pesqui. 2010;17(4):209-213. http://dx.doi.org/10.1590/ S1809-29502010000300004.

35. Lin S, McElfresh J, Hall B, Bloom R, Farrell K. Inspiratory muscle training in patients with heart failure: a systematic review. Cardiopulm Phys Ther J. 2012;23(3):29-36. http://dx.doi. org/10.1097/01823246-201223030-00005. PMid:22993500.

36. Menezes KKP, Nascimento LR, Polese JC, Ada L, TeixeiraSalmela LF. Effect of high-intensity home-based respiratory muscle training on strength of respiratory muscles following a stroke: a protocol for a randomized controlled trial. Braz J Phys Ther. 2017;21(5):372-7. http://dx.doi.org/10.1016/j. bjpt.2017.06.017. PMid:28728960.

37. Carvalho VO, Guimarães GC, Carrara D, Bacal F, Bocchi EA Validação da versão em português do Minnesota Living with Heart Failure Questionnaire. Arq Bras Cardiol. 2009;93(1):3944. http://dx.doi.org/10.1590/S0066-782X2009000700008. PMid:19838469.

38. Ciconelli RM, Ferraz MB, Santos W, Meinão I, Quaresma MR. Tradução para a língua portuguesa e validação do questionário genérico de avaliação de qualidade de vida SF-36 (Brasil SF-36). Rev Bras Reumatol. 1999;39(3):143-50.

39. Evans A, Watson K, Hawkins M, Cowley AJ, Johnston ID, Kinnear WJ. Respiratory muscle strength in chronic heart failure. Thorax. 1995;50(6):625-628. http://dx.doi.org/10.1136/ thx.50.6.625.
40. Meyer FJ, Borst MM, Zugck C, Kirschke A, Schellberg $D$, Kubler $W$, et al. Respiratory muscle dysfunction in congestive heart failure: clinical correlation and prognostic significance. Circulation. 2001;103(17):2153-8. http://dx.doi. org/10.1161/01.CIR.103.17.2153. PMid:11331255.

41. Forgiarini LA Jr, Rubleski A, Garcia D, Tieppo J, Vercelino R. Avaliação da força muscular respiratória e da função pulmonar em pacientes com insuficiência cardíaca. Arq Bras Cardiol. 2007;89(1):36-41. http://dx.doi.org/10.1590/ S0066-782X2007001300007. PMid:17768581.

42. Gomes M No, Ferrari F, Helal L, Lopes AA, Carvalho VO, Stein $R$. The impact of high-intensity inspiratory muscle training on exercise capacity and inspiratory muscle strength in heart failure with reduced ejection fraction: a systematic review and meta-analysis. Clin Rehabil. 2018;32(11):1482-92. http:// dx.doi.org/10.1177/0269215518784345. PMid:29984598.

43. Katayama K, Iwamoto E, Ishida K, Koike T, Saito M. Inspiratory muscle fatigue increases sympathetic vasomotor outflow and blood pressure during submaximal exercise. Am J Physiol. 2012;302(10):R1167-75. http://dx.doi.org/10.1152/ ajpregu.00006.2012. PMid:22461178.

44. Dempsey JA. New perspectives concerning feedback influences on cardiorespiratory control during rhythmic exercise and on exercise performance. J Physiol. 2012;590(17):4129-44. http://dx.doi.org/10.1113/ jphysiol.2012.233908. PMid:22826128.

45. Piepoli MF, Spoletini I, Rosano G. Monitoring functional capacity in heart failure. Eur Heart J Suppl. 2019;21(suppl. M):M9-M12. http://dx.doi.org/10.1093/eurheartj/suz216.

46. Awotidebe TO, Adeyeye VO, Adedoyin RA, Ogunyemi SA, Oke RL, Ativie RN, et al. Assessment of functional capacity and sleep quality of patients with chronic heart failure. Hong Kong Physiother J. 2016;36:17-24. http://dx.doi. org/10.1016/j.hkpj.2016.10.001. PMid:30931035.

47. Silva ES, Santos-de-Araújo AD, Silva TS, Lira JLF, Calles ACN, Bassi D. Correlação entre força muscular respiratória, capacidade funcional e qualidade de vida na insuficiência cardíaca congestiva. ConScientiae Saúde. 2019;18(2):249-54. http://dx.doi.org/10.5585/conssaude.v18n2.11401.

48. Pereira DAG, Rodrigues RS, Samora GAR, Lage SM, Alencar MCN, Parreira VF, et al. Capacidade funcional de indivíduos com insuficiência cardíaca avaliada pelo teste de esforço cardiopulmonar e classificação da New York Heart Association. Fisioter Pesq. 2012;19(1):52-56. http://dx.doi.org/10.1590/ S1809-29502012000100010.

49. Witt JD, Guenette JA, Rupert JL, Mckenzie D, Sheel AW. Inspiratory muscle training attenuates the human respiratory muscle metaboreflex. J Physiol. 2007;548(3):1019-1028.

50. Jorge AJL, Rosa MLG, Correia DMS, Martins WA, Ceron DMM, Coelho LCF, et al. Avaliação da qualidade de vida em pacientes com e sem insuficiência cardíaca na atenção primária. Arq Bras Cardiol. 2017;109(3):248-52. http://dx.doi. org/10.5935/abc.20170123. PMid:28832746.

51. Zaponi RS, Fres AR, Mora CTR, Ruaro JA, Daniel CR. Avaliação da qualidade de vida de portadores de insuficiência cardíaca congestiva e sua correlação com a Classificação Internacional de Funcionalidade, Incapacidade e Saúde. Acta Fisiat. 2015;22(3):105-110. http://dx.doi.org/10.5935/01047795.20150021. 